\title{
GENES REgulating PROGRAMMED CELL DEATH ARE SIGNIFICANTLY UPREGULATED IN PORCINE IMMATURE OOCYTES
}

Katarzyna Stefańska ${ }^{1}$, Małgorzata Józkowiak², Paweł Antosik ${ }^{3}$, Dorota Bukowska ${ }^{3}$, Piotr Celichowski ${ }^{1}$, Małgorzata Bruska ${ }^{4}$, Michał Nowicki ${ }^{1}$, Bartosz Kempisty ${ }^{1,4,5}$, Jana Zakova ${ }^{5}$, Marie Machatkova $^{6}$, Michal Jeseta ${ }^{5}$

\begin{abstract}
Correct maturation of the oocyte is crucial for further fertilization and embryogenesis. It comprises of both nuclear and cytoplasmic maturation, during which the proteins, nutrients and mRNAs are assembled. Cumulus cells are connected with the oocyte via gap-junctions, which enable bi-directional transfer of molecules, forming cumulus-oocyte complex (COC). The expression pattern in CCs is thought to resemble the genes expressed in the oocyte. The CCs surrounding the gamete of high developmental competence have an increased expression of apoptotic markers. Therefore, our aim in this study was to determine whether any apoptosis-related genes are upregulated in porcine oocytes before or after IVM. We isolated COCs from 45 pubertal crossbred gilts, performed brilliant cresyl blue (BCB) staining and analyzed the gene expression pattern in oocytes before and after IVM with the use of microarray analysis. The results include 419 differentially expressed transcripts, 25 of which belong to „regulation of apoptosis” and „regulation of cell death" GO BP terms. This set of genes includes BCLAF1, EIF2AK3, KLF10, MIF, MAP3K1, NOTCH2, TXNIP and $A P P$, all of which have been upregulated in immature porcine oocytes. Our results suggest that they play part in porcine oocyte maturation and could be used as potential markers of female gamete's developmental competence. This knowledge could serve as a basis to improve ART in pigs.
\end{abstract}

Running title: Differential expression of programmed cell death genes in porcine oocytes

Keywords: pig, oocytes, IVM, microarray, programmed cell death

\footnotetext{
${ }^{1}$ Department of Histology and Embryology, Poznan University of Medical Sciences, Poznan, Poland

${ }^{2}$ Department of Toxicology, Poznan University of Medical Sciences, Poznan, Poland

${ }^{3}$ Veterinary Center, Nicolaus Copernicus University in Torun, Toruń, Poland

${ }^{4}$ Department of Anatomy, Poznan University of Medical Sciences, Poznan, Poland

${ }^{5}$ Department of Obstetrics and Gynecology, University Hospital and Masaryk University, Brno, Czech Republic

${ }^{6}$ Veterinary Research Institute, Brno, Czech Republic

* Correspondence: e-mail: jeseta@gmail.com

Full list of author information is available at the end of article
} 


\section{Introduction}

Mammalian oogenesis begins during the fetal life, when primary oocytes in primordial follicles are produced. Female gamete development is arrested in prophase I of meiotic division, specifically in diplotene. Further meiotic progression and oocyte nuclear maturation occurs after puberty, prior to ovulation, in response to gonadotropins, and halts at metaphase II. After fertilization, the meiotic division completes [1,2]. For full oocyte maturity, the cytoplasmic maturation must occur as well. It can be defined as reorganization of organelle and cytoskeleton and assembly of proteins, nutrients and mRNAs necessary for embryo's initial development [2-4]. Thus, correct oocyte maturation is essential for embryogenesis and successful pregnancy.

In the antral ovarian follicle, the oocyte remains in close contact with surrounding follicular somatic cells, which form the cumulus oophorus [5]. Cumulus cells (CCs) are connected with the oocyte via gap junctions, which enable bi-directional transfer of small weight molecules, such as ions, metabolites and amino acids, as well as the RNA [6]. Both gap junction communication and paracrine signaling in COC (cumulus-oocyte complex) play vital roles in oocyte's development, as well as proper function of the CCs $[3,7]$. The oocyte regulates folliculogenesis by influencing follicular cell differentiation, proliferation, expansion and apoptosis via oocyte secreted factors (OSF) [8]. CCs, on the other hand, are crucial in oocyte's nuclear and cytoplasmic maturation $[6,9]$ and protect it from apoptosis caused by oxidative stress [10]. The studies in mice have also shown that contact between oocytes and CCs is important for gamete spindle positioning and developmental competence during the maturation process [11]. Transcriptomic studies in cattle aimed to identify genes expressed in both oocytes and cumulus cells during the process of gamete's maturation [9]. As a result, the molecular crosstalk between both cell types has been indicated.

Since the assisted reproduction technology (ART) in pigs remains less efficient than in other species, there is a constant need for its improvement [12]. There is a possibility to recover oocytes destined for atresia to improve livestock production system. However, such gametes remain immature, therefore a proper in vitro maturation technique (IVM) must be utilized to obtain an embryo, as the quality of the oocyte is crucial for its ability to develop into a blastocyst [13].

Several approaches may be taken to determine immature oocyte's developmental competence, such as utilizing gene expression patterns in follicular cells (cumulus cells and granulosa cells) as predictors of gamete's quality [14]. Another possible approach is to examine the transcriptome of the oocyte itself, before and after IVM, and such approach has been undertaken in this study.
One of the processes that is thought to affect developmental competence of the oocyte is apoptosis. Thus, there have been some studies conducted to determine whether it's occurrence in CCs influences gamete quality. The study in humans indicated that there is a correlation between oocyte's competence and an increase in apoptotic markers in surrounding CCs [15]. Similar results were obtained in the cattle [16].

In this study we focused on porcine oocyte gene expression analysis before and after IVM with the use of microarray approach, in order to determine whether there are differences in expression of apoptosis-related genes, dependent on maturity stage of the female gamete. Indeed, we have observed downregulation of several genes involved in apoptosis after IVM, indicating that they may be potential markers of oocyte's competence.

\section{Material and Methods Animals}

A total of 45 pubertal crossbred Landrace gilts bred on a local, commercial farm were used in this study. They had a mean age of 155 days (range 140-170 days) and weight of $100 \mathrm{~kg}$ (95-120 kg). All of the animals were housed under identical conditions and fed the same forage (depending on age and reproductive status). The experiments were approved by the Poznań University of Medical Sciences Bioethical Committee (Resolution 32/2012).

\section{Collection of porcine ovaries and COCs}

Ovaries and reproductive tracts were recovered at slaughter and transported to the laboratory at $38^{\circ} \mathrm{C}$ in $0.9 \% \mathrm{NaCl}$ within $40 \mathrm{~min}$. To provide optimal conditions for subsequent oocyte maturation and fertilization in vitro, the ovaries of each animal were placed in 5\% fetal bovine serum solution (Sigma-Aldrich Co., St. Louis, MO) in phosphate-buffered saline (PBS). Then, single large follicles $(>5$ $\mathrm{mm}$ ) were opened by puncturing with a $5 \mathrm{~mL}$ syringe and 20-G needle in a sterile petri dish, and COCs were recovered. The COCs were washed three times in modified PBS supplemented with $36 \mathrm{mg} /$ $\mathrm{mL}$ pyruvate, $50 \mathrm{mg} / \mathrm{mL}$ gentamycin, and $0.5 \mathrm{mg} /$ $\mathrm{mL}$ bovine serum albumin (BSA; Sigma-Aldrich). COCs were selected under an inverted microscope Zeiss, Axiovert 35 (Lübeck, Germany), counted and morphologically evaluated using the scale suggested by de Looset al. (1991). Only COCs of grade I with homogeneous ooplasm and uniform, compact CCs were considered for the following steps of the experiment, resulting in 300 grade I oocytes $(3 \times \mathrm{n}=50$ before IVM, 3 x n=50 after IVM).

\section{Assessment of oocyte developmental competence by brilliant cresyl blue test}

To perform the brilliant cresyl blue (BCB) staining test, oocytes were washed twice in modified 
Dulbecco PBS (DPBS; Sigma-Aldrich) supplemented with $50 \mathrm{IU} / \mathrm{mL}$ penicillin, $50 \mathrm{mg} / \mathrm{mL}$ streptomycin (Sigma-Aldrich), 0.4\% BSA (w/v), $0.34 \mathrm{mM}$ pyruvate, and $5.5 \mathrm{mM}$ glucose Dulbecco's phosphate buffered saline modified (DPBSm). Thereafter, they were treated with $13 \mathrm{mM}$ BCB (Sigma-Aldrich) diluted in DPBSm at $38.5^{\circ} \mathrm{C}$ and $5 \% \mathrm{CO} 2$ for $90 \mathrm{~min}$. After treatment, the oocytes were transferred to DPBSm and washed twice. During the washing procedure, the oocytes were examined under an inverted microscope and classified as either stained blue $(\mathrm{BCB}+)$ or colourless (BCB-). The collected pool comprised $55 \%$ of $\mathrm{BCB}+$ and $45 \%$ of BCB- oocytes. Immature oocytes have compact $\mathrm{CC}$ layers that require removal for further oocyte evaluation. Hence, the BCB+ COCs were first incubated with bovine testicular hyaluronidase (Sigma-Aldrich) for $2 \mathrm{~min}$ at $38^{\circ} \mathrm{C}$ to separate CCs and GCs. Cells were then removed by vortexing the $\mathrm{BCB}+$ oocytes in $1 \%$ sodium citrate buffer followed by mechanical displacement using a small-diameter glass micropipette. Only the GC-free BCB+ oocytes were used for subsequent IVM and microarray analysis.

\section{IVM of porcine COCs}

After the first BCB test, the COCs with stained blue cytoplasm $(\mathrm{BCB}+)$ were cultured in $\operatorname{Nunclon}^{\mathrm{TM}} \Delta$ four-well dishes in $500 \mathrm{~mL}$ of standard porcine IVM culture medium TCM-199 (tissue culture medium) with Earle's salts and l-glutamine (Gibco BRL Life Technologies, Grand Island, NY) supplemented with $2.2 \mathrm{mg} / \mathrm{mL}$ sodium bicarbonate (Nacalai Tesque, Inc., Kyoto, Japan), $0.1 \mathrm{mg} / \mathrm{mL}$ sodium pyruvate (Sigma-Aldrich), $10 \mathrm{mg} / \mathrm{mL}$ BSA (Sigma-Aldrich), $0.1 \mathrm{mg} / \mathrm{mL}$ cysteine (Sigma-Aldrich), 10\% filtered porcine follicular fluid ( $\mathrm{v} / \mathrm{v})$, and gonadotropin supplements at final concentrations of $2.5 \mathrm{IU} / \mathrm{mL}$ human chorionic gonadotropin (Ayerst Laboratories, Inc., Philadelphia, PA) and $2.5 \mathrm{IU} / \mathrm{mL}$ equine chorionic gonadotropin (Intervet, Whitby, Canada). Wells were covered with a mineral oil overlay and cultured for $44 \mathrm{~h}$ at $38^{\circ} \mathrm{C}$ under $5 \% \mathrm{CO} 2$. After cultivation, the BCB staining test was performed again, and $\mathrm{BCB}+$ oocytes were used for further experiments.

\section{RNA extraction from porcine oocytes}

Oocytes investigated before and after IVM were pooled into three independent samples for each experimental group. Total RNA was extracted from samples using TRI Reagent (Sigma, St. Louis, MO) and RNeasy MinElute cleanup kit (Qiagen, Hilden, Germany). The amount of total mRNA was determined from the optical density (OD) at $260 \mathrm{~nm}$, and the RNA purity was estimated using the 260/280 $\mathrm{nm}$ absorption ratio (higher than 1.8) (NanoDrop spectrophotometer; Thermo Scientific, ALAB, Poland). The RNA integrity and quality were checked on a Bioanalyzer 2100 (Agilent Technologies, Inc., Santa Clara, CA). The resulting RNA integrity num- bers were between 8.5 and 10 with an average of 9.2 (Agilent Technologies, Inc.). The RNA in each sample was diluted to a concentration of $100 \mathrm{ng} / \mathrm{mL}$ with an OD260/0D280 ratio of 1.8/2.0. From each RNA sample, 500 ng of RNA was taken for microarray expression assays.

\section{Microarray expression analysis and statistics}

Experiments were performed in three replicates. Total RNA (100 ng) from each pooled sample was subjected to two round sense cDNA amplification (Ambion® WT Expression Kit). The obtained cDNA was used for biotin labeling and fragmentation using Affymetrix GeneChip® WT Terminal Labeling and Hybridization (Affymetrix). Biotin-labeled fragments of cDNA ( $5.5 \mu \mathrm{g}$ ) were hybridized to the Affymetrix ${ }^{\circledR}$ Porcine Gene 1.1 ST Array Strip $\left(48^{\circ} \mathrm{C} / 20\right.$ h). Then, microarrays were washed and stained according to the technical protocol, using Affymetrix GeneAtlas Fluidics Station. The array strips were scanned using the Imaging Station of the GeneAtlas System. The preliminary analysis of the scanned chips was performed using Affymetrix GeneAtlas ${ }^{\mathrm{TM}}$ Operating Software. Quality of gene expression data was checked according to quality control criteria provided by the software. Obtained CEL files were imported into downstream data analysis software.

All analyzes were performed using BioConductor software, based on the statistical R programming language. For background correction, normalization and summation of raw data, the Robust Multiarray Averaging (RMA) algorithm implemented in "affy" package of BioConductor was applied. Biological annotation was taken from the BioConductor "oligo" package where annotated data frame object was merged with normalized data set, leading to a complete gene data table. Statistical significance of analyzed genes was performed by moderated t-statistics from the empirical Bayes method. Obtained $p$ value was corrected for multiple comparisons using the Benjamini and Hochberg's false discovery rate. The selection of significantly changed gene expression was based on p value beneath 0.05 and expression fold higher than $|2|$.

Functional annotation clustering of differentially expressed genes was performed using DAVID (Database for Annotation, Visualization and Integrated Discovery). Gene symbols for up- or down-regulated genes from each of the compared groups were loaded to DAVID by "RDAVIDWebService" BioConductor package. For further analysis we have chosen the enriched GO terms which contained at least 5 genes and exhibited a p.value (Benjamini) lower than 0.05 . The enriched G0 terms were subjected to hierarchical clusterization algorithm and presented as a heatmaps.

Subsequently we analyzed the relation between the genes belonging to chosen GO terms with the GOplot package [17]. The GoPlot package calculat- 
ed the z-score: the number of up-regulated genes minus the number of down- regulated genes divided by the square root of the count. This information allowed to estimate the change course of each gene-ontology term.

Interactions between chosen differentially expressed genes/proteins belonging to chosen ontology group were investigated using the STRING10 software (Search Tool for the Retrieval of Interacting Genes). List of gene names were used as query for interaction prediction. Searching criteria based on co-occurrences of genes/proteins in scientific texts (text mining), co-expression and experimentally observed interactions. The results of such analysis generated gene/protein interaction network where the intensity of the edges reflects the strength of interaction score. Besides interaction prediction, STRING also allowed us to perform functional enrichments of GO terms based on previously uploaded gene sets.

Finally, the functional interactions between genes that belongs to the chosen GO BP terms were investigated by REACTOME FIViz application to the Cytoscape 3.6.0 software. The ReactomeFIViz app is designed to find pathways and network patterns related to cancer and other types of disease. This app accesses the pathways stored in the Reactome database, allowing to perform pathway enrichment analysis for a set of genes, visualize hit pathways using manually laid-out pathway diagrams directly in Cytoscape, and investigate functional relationships among genes in hit pathways. The app can also access the Reactome Functional Interaction (FI) network, a highly reliable, manually curated pathway-based protein functional interaction network covering over $60 \%$ of human proteins.

\section{Ethical approval}

The research related to animal use has been complied with all the relevant national regulations and instructional policies for the care and use of animals. Bioethical Committee approval no. 32/2012.

\section{Results}

Whole transcriptome profiling with the use of Affymetrix microarrays allowed us to analyze the gene expression changes in freshly isolated oocytes, before in vitro procedure ("before IVM"), in relation to after in vitro maturation ("after IVM"). Using Affymetrix ${ }^{\circledR}$ Porcine Gene 1.1 ST Array, we have examined the expression of 12258 porcine transcripts. Genes with fold change higher than $|2|$ and with corrected $p$ value lower than 0.05 were considered as differentially expressed. This set of genes consisted of 419 different transcripts. Subsequently, the genes were used for identification of significantly enriched GO BP terms.

DAVID (Database for Annotation, Visualization and Integrated Discovery) software was used for extraction of the genes belonging to Gene Ontology Biological Process terms (GO BP). In this paper, we focused on "regulation of cell death" and "regulation of apoptosis" GO BP terms. We found that 25 genes from these GO BP terms were significantly represented in down-regulated gene sets. These sets of genes were subjected to hierarchical clusterization procedure and presented as heat maps (Fig. 1). regulation of apoptosis

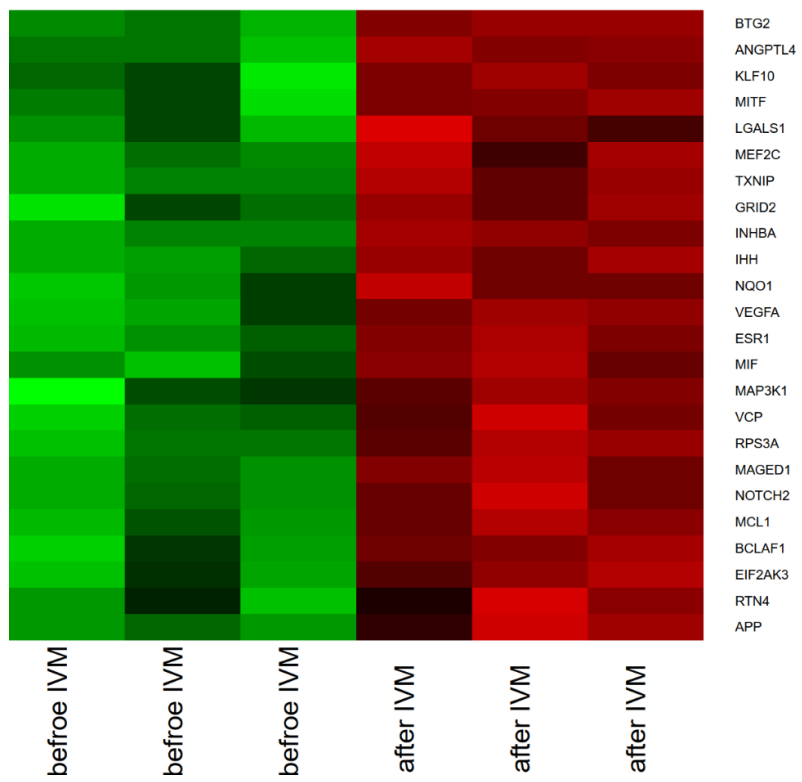

regulation of cell death

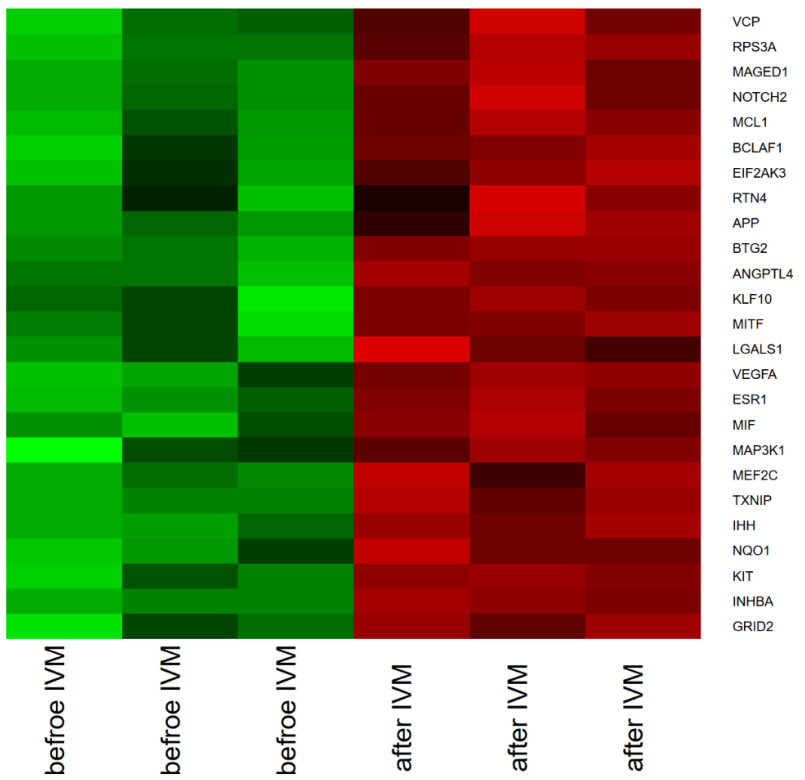

FIGURE 1 Heat map representations of differentially expressed genes belonging to the "regulation of cell death", "regulation of apoptosis" GO BP terms. Arbitrary signal intensity acquired from microarray analysis is represented by colours (green, higher; red, lower expression). Log2 signal intensity values for any single gene were resized to Row Z-Score scale (from -2 , the lowest expression to +2 , the highest expression for single gene) 
TABLE 1 Gene symbols, fold changes in expression, corrected p values and LogFC of studied genes

\begin{tabular}{l|c|c|c}
\hline GENE SYMBOL & FOLD CHANGE & ADJUSTED P. VALUE & ENTREZ GENE ID \\
\hline VEGFA & 0,069689 & 0,001913 & 397157 \\
\hline BTG2 & 0,074386 & $9,55 \mathrm{E}-05$ & $1 \mathrm{E}+08$ \\
\hline ESR1 & 0,08163 & 0,000522 & 397435 \\
\hline ANGPTL4 & 0,183631 & 0,000513 & 397628 \\
\hline RTN4 & 0,231378 & 0,027496 & $1 \mathrm{E}+08$ \\
\hline INHBA & 0,24126 & 0,000148 & 397093 \\
\hline MCL1 & 0,24418 & 0,001775 & 397648 \\
\hline LGALS1 & 0,269317 & 0,01029 & 414915 \\
\hline IHH & 0,304996 & 0,000551 & 397174 \\
\hline MAGED1 & 0,305616 & 0,000706 & 414852 \\
\hline NQ01 & 0,306986 & 0,0045 & $1 \mathrm{E}+08$ \\
\hline APP & 0,324139 & 0,005602 & 397663 \\
\hline TXNIP & 0,355539 & 0,000781 & 733688 \\
\hline MAP3K1 & 0,368765 & 0,024748 & 396617 \\
\hline MIF & 0,375718 & 0,002882 & 397412 \\
\hline NOTCH2 & 0,384826 & 0,002524 & $1 \mathrm{E}+08$ \\
\hline KLF10 & 0,405439 & 0,006845 & $1 \mathrm{E}+08$ \\
\hline EIF2AK3 & 0,41889 & 0,008422 & $1,01 \mathrm{E}+08$ \\
\hline BCLAF1 & 0,419563 & 0,005984 & $1 \mathrm{E}+08$ \\
\hline KIT & 0,430444 & 0,002556 & 396810 \\
\hline VCP & 0,435612 & 0,007402 & 397524 \\
\hline GRID2 & 0,444289 & 0,008595 & $1,01 \mathrm{E}+08$ \\
\hline MEF2C & 0,453593 & 0,003964 & 733590 \\
\hline RPS3A & 0,462721 & 0,00262 & 414390 \\
\hline MITF & 0,49166 & 0,00633 & 414902 \\
\hline
\end{tabular}
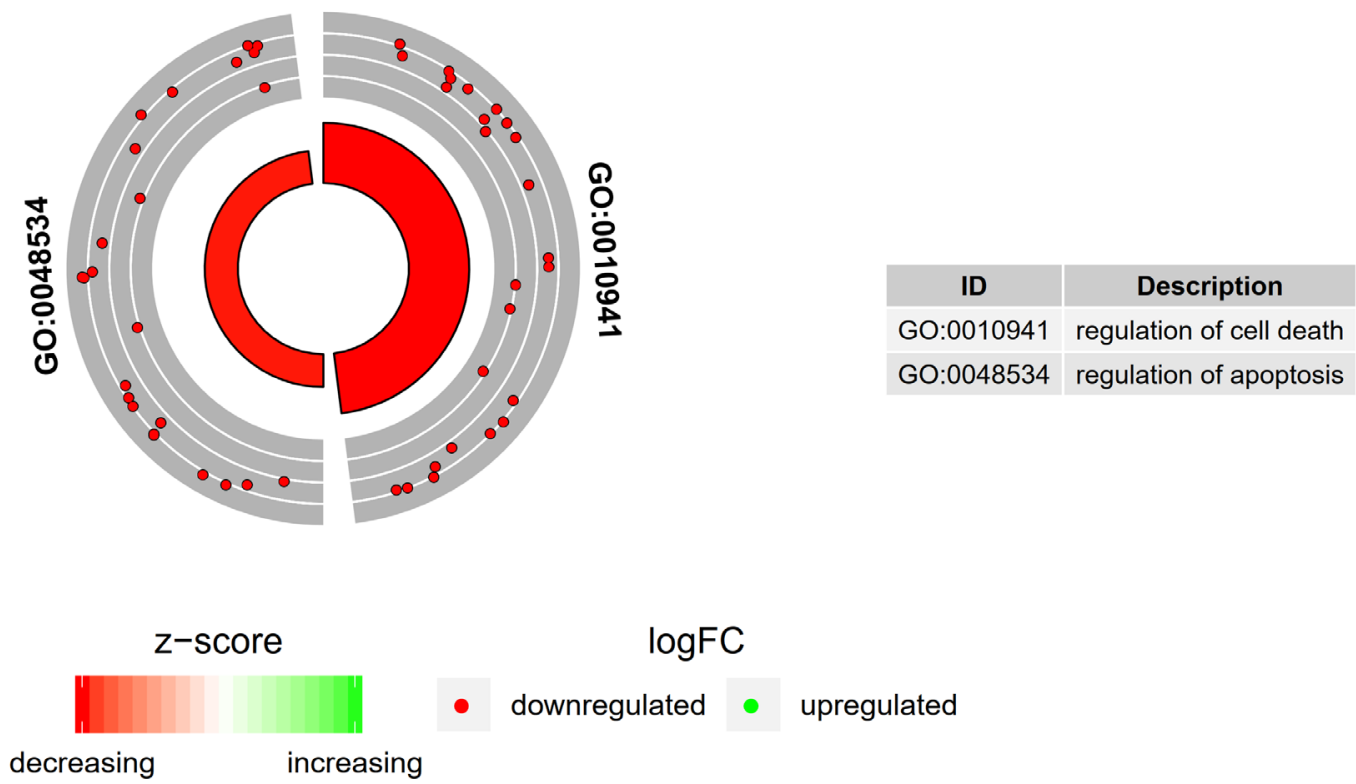

FIGURE 2 The circle plot showing the differently expressed genes and z-scores "regulation of cell death", "regulation of apoptosis" GO BP terms. The outer circle shows a scatter plot for each term of the fold change of the assigned genes. Green circles display up- regulation and red ones down- regulation. The inner circle shows the z-score of each GO BP term. The width of each bar corresponds to the number of genes within GO BP term and the color corresponds to the z-score 


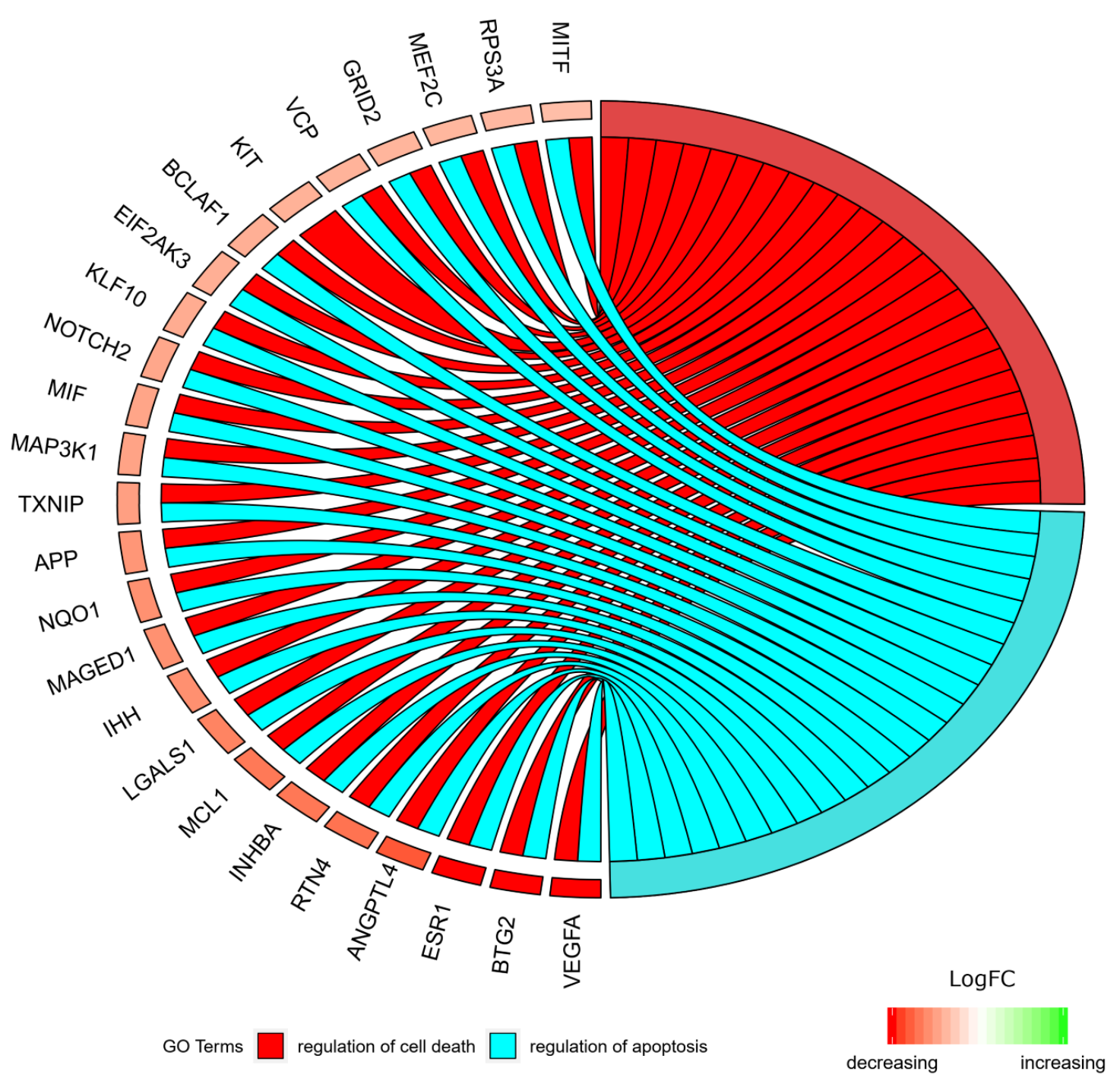

FIGURE 3 The representation of the mutual relationship between differently expressed genes that belongs to the "regulation of cell death", "regulation of apoptosis" GO BP terms. The ribbons indicate which gene belongs to which categories. The middle circle represents logarithm from fold change (LogFC). The genes were sorted by logFC from most to least changed gene. The color of the each LogFC bar corresponds with LogFC value

Set of the differentially expressed genes belonging to "regulation of cell death" and "regulation of apoptosis" GO BP terms, with their official gene symbols, fold changes in expression, corrected $p$ values and $\log \mathrm{FC}$, was shown in table 1.

The enrichment of each GO BP term as well KEGG pathway was calculated as a z-score and shown on the circle diagram (Fig. 2).

Moreover, in Gene Ontology database, genes that formed one particular GO group can also belong to other different GO term categories. By this reason we explore the gene intersections between selected GO BP terms. The relation between those GO BP terms was presented as circle plot (Fig. 3).

STRING-generated interaction network was created with differentially expressed genes belonging to the "fatty acid metabolic process" ontology group. The intensity of the edges reflects the strength of in- teraction score (Fig. 4). Finally, we investigated the functional interactions between chosen genes with REACTOME FIViz app to Cytoscape 3.6.0 software. The results were shown in (Fig. 5).

\section{Discussion}

Apoptosis is a physiological process necessary for maintaining homeostasis in the organism, with its occurrence previously described in cumulus and granulosa cells surrounding the mammalian oocyte $[15,16]$, as well as in oocytes themselves [18]. It involves activation of, among others, the $\mathrm{Bcl}-2$ family of proteins and the caspases [19]. The aim of this study was to examine whether any apoptotic markers can be found in porcine oocyte, either immature or after IVM. We found 419 differentially expressed transcripts in oocytes before and after IVM, 25 of which belong to "regulation of apoptosis" and "regulation of 


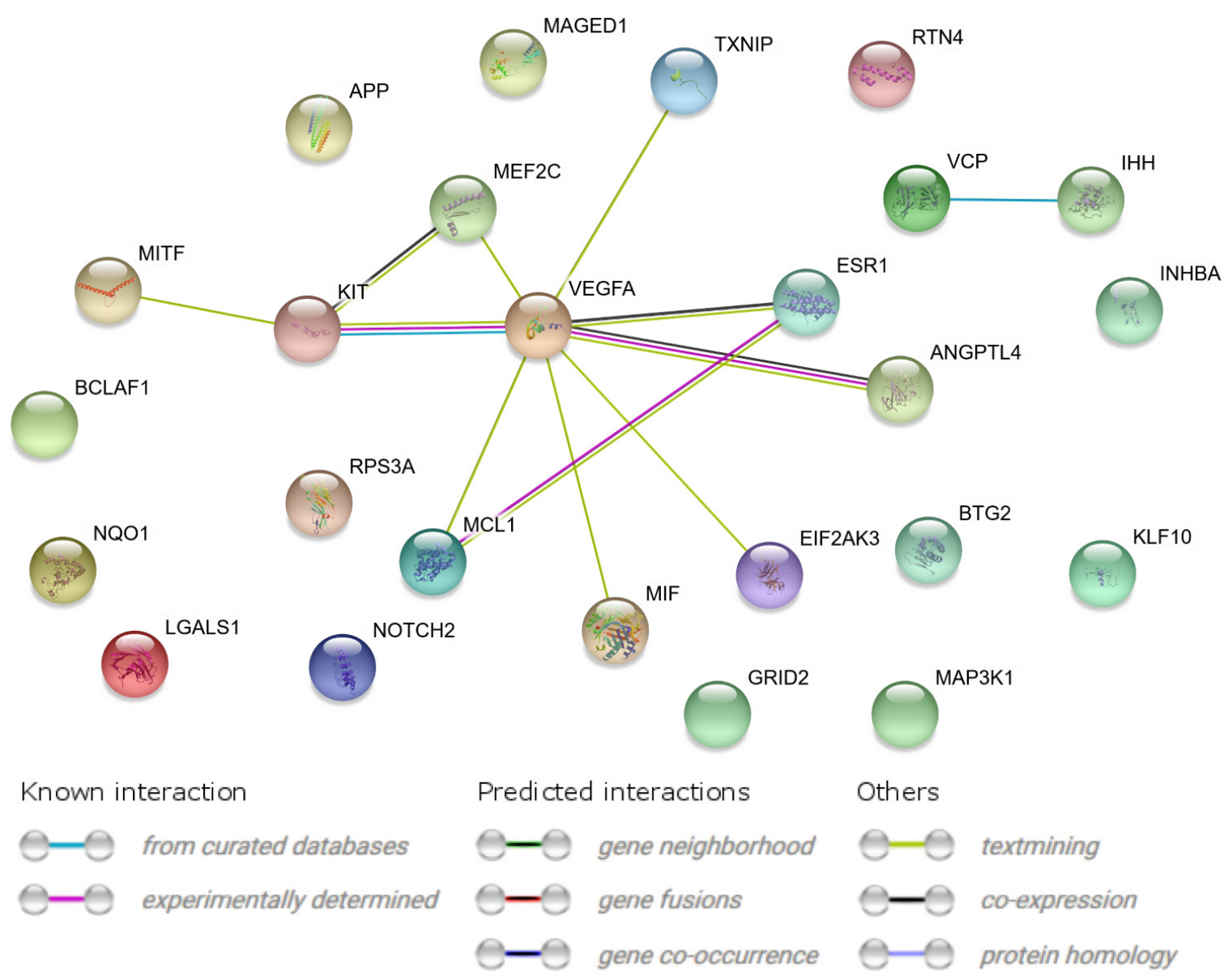

FIGURE 4 STRING-generated interaction network between genes that belongs to the "regulation of cell death", "regulation of apoptosis" GO BP terms. The intensity of the edges reflects the strength of interaction score

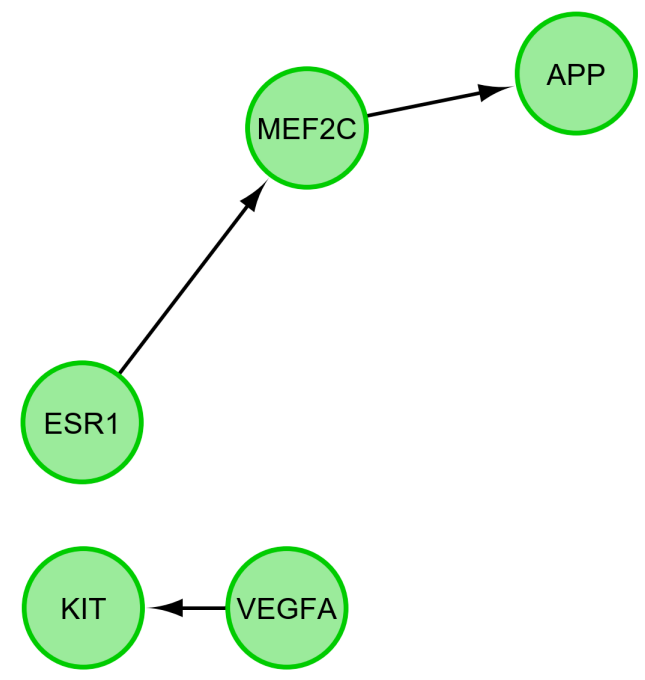

FIGURE 5 Functional interaction (FI) between differently expressed genes that belongs to "regulation of cell death", "regulation of apoptosis" GO BP terms. In following figure "->" stands for activating/catalyzing, "-|" for inhibition, "-" for FIs extracted from complexes or inputs, and "---" for predicted FIs

cell death" GO BP terms. All of those 25 genes were significantly upregulated before IVM, with particular focus in this work placed on BCLAF1, EIF2AK3, KLF10, MIF, MAP3K1, NOTCH2, TXNIP and APP.
BCLAF1 (BCL2-associated transcription factor 1) belongs to both "regulation of apoptosis" and "regulation of cell death" GO BP terms and was significantly upregulated in immature oocytes. It acts as 
a transcriptional repressor and it's interaction with BCL2 and BCLXL proteins has been described by Kasof et al. [20]. Both BCL2 and BCXL families have antiapoptotic properties, association with BCLAF1 results in repression of their transcription. This suggests BCLAF1's role in apoptosis promotion. However, it's involvement in apoptosis hasn't been described in porcine oocytes yet.

Another transcription factor that was upregulated in porcine oocytes before IVM is KLF10 (Kruppel-like factor 10), also called TIEG (TGF-beta inducible early gene), containing a three-zinc finger domain. Its expression has primarily been described in human osteoblast cells and subsequent studies have revealed its presence in pancreas, placenta, heart, ovary and peripheral blood leukocytes [21]. Studies conducted by Tachibana et al. have shown that TGF-beta regulates the expression of KLF10. Moreover, the role of this transcription factor in apoptosis has been well documented in exocrine pancreatic epithelial cells, where its overexpression resulted in cell death [22].

EIF2AK3 (Eukaryotic translation initiation factor 2 -alpha kinase 3 ) is a regulator of protein synthesis initially described in rats. Its expression occurs especially in the pancreas $[23,24]$. It phosphorylates eIF2-alpha (Eukaryotic translation initiation factor 2-aplha), therefore inhibits the translation process, and is involved in mitotic spindle formation during cell division [25]. EIF2AK3 also plays a role in adapting to ER stress by decreasing protein production. However, when that stress is not alleviated, EIF2AK3 indirectly causes apoptosis induction [26]. Therefore, EIF2AK3 upregulation in immature oocyte may suggest that the ER stress occurs during the IVM process.

MIF (Macrophage migration inhibitory factor) is a cytokine that activates macrophages and is expressed at the sites of inflammation. Apart from that, it is also suspected that it plays a role in the establishment of pregnancy, as the studies in mice have shown that it is expressed in the ovary, the oviduct and the uterus, as well as in the oocyte and an early embryo [27]. Wada et al. have examined the expression of MIF in human ovary and found its mRNA in follicular fluid and granulosa cells, suggesting involvement in oocyte development [28]. Our results are consistent with aforementioned studies and show that MIF is upregulated in immature porcine oocytes and may be a potential marker for the female gamete maturation.

Another upregulated gene in oocytes before IVM was NOTCH2, which encodes a transmembrane receptor involved in NOTCH signalling pathway, which plays a role in gene expression regulation during many cellular processes such as proliferation, differentiation and apoptosis. This pathway's activation is dependent on direct contact between neighbouring cells. According to the study conduct- ed by Johnson et al., Notch2 is expressed in murine cumulus and mural granulosa cells, but is not present in oocytes [29]. NOTCH signalling pathway is proven to play a critical role in follicle development and granulosa cells proliferation, as its inhibition results in detachment of granulosa cells and oocyte degeneration in mice [30]. In vivo studies in mice have shown that Notch2 conditional deletion results in an increased number of oocytes, because of decreased oocyte apoptosis [31]. There have not been similar studies performed in pigs, however our results indicate either that NOTCH2 is involved in oocyte maturation or its mRNA has been transferred from surrounding somatic cells.

MAP3K1 (Mitogen-activated protein kinase kinase kinase 1), otherwise called $M E K K 1$, encodes a protein that activates the JNK and ERK, as well as NFkB and p300 pathways. It acts as an apoptotic regulator, either generating the anti-apoptotic signals as a full length protein, or pro-apoptotic signals after caspase-mediated cleavage [32]. The role of other mitogen-activated protein kinase, MAPK-activated protein kinase 2 (MK2), has already been described in porcine oocytes by $\mathrm{Ou}$ et al. It was shown, that MK2 affects CCs expansion and oocyte meiotic maturation by spindle organization and chromosome aignment regulation [33]. Considering MAP3K1 upregulation in porcine oocytes before IVM, it is possible that it is also involved in gamete's maturation and may serve as a marker of developmental competence.

In present study, we have also observed the upregulation of TXNIP (Thioredoxin-interacting protein) in immature porcine oocytes. Results obtained by Junn et al. suggest that this protein is an important regulator of oxidative stress response via thioredoxin activity inhibition [34], which was later confirmed by Wang et al. in rat cardiomyocytes, resulting in an increased apoptosis [35]. TXNIP expression during oocyte maturation has already been examined in cows. This study, like the one described in this paper, utilized microarray transcriptomic analysis. However, the examined cells were CCs enclosing in vitro and in vivo matured oocytes. The results have shown a decreased expression of TXNIP in IVM CCs and more than twofold increase in apoptosis [36]. Another study, conducted in murine oocytes by Lee et al., has clearly shown that Txnip was upregulated in immature oocytes and its depletion resulted in maturation and meiosis arrest. Moreover, this protein's critical role in glucose metabolism and cytoplasmic streaming in female gametes has been indicated [37].

APP (Amyloid beta A4 precursor protein) contributes to amyloid plaque formation in patients with Alzheimer's disease and Down syndrome. However, it is expressed not only in the brain, but in other tissues as well. Its role in cell adhesion has also been reported. Studies conducted by Fisher et 
al. have shown that $A P P$ mRNA is present in murine oocytes and in the two-cell stage embryo, suggesting that it is one of the first genes transcribed in developing embryo [38]. Moreover, Kimura et al. reported presence of $A P P$ mRNA in porcine granulosa cells from small and large follicles and soluble form of this protein in the folliular fluid [39]. The results of another study, conducted in cows, indicate that $A P P$, among other genes, can be a helpful in oocyte developmental competence predictor [40]. However, our results describe an increase in expression of $A P P$ in porcine oocytes before IVM.

In conclusion, all the aforementioned genes linked with apoptosis have been upregulated in porcine oocytes before IVM. There is a possibility that they could be used as potential markers of female gamete's developmental competence and play part in porcine oocyte maturation. The transcripts may have been accumulated in the oocyte during its cytoplasmic maturation could reflect mRNAs present in CCs, as the gap-junction mediated transfer of molecules in COCs has been well documented. Such markers may be helpful in selection of good quality gametes and porcine ART optimization.

\section{Acknowledgements}

This publication and its results are an outcome of a cooperation between Poznan University of Medical Sciences (Poznań, Poland) and Polish Ministry of Science and Higher Education, with Institute of Advanced Sciences Sp. z o.o. (Poznań, Poland), as a part of the "Professional $\mathrm{PhD}$ " programme.

\section{Corresponding author}

Michal Jeseta, Department of Obstetrics and Gynecology, University Hospital and Masaryk University, Obilní trh 526/11, 60200 Brno, Czech Republic. Tel.: +420 53223 8236, e-mail: jeseta@ gmail.com.

\section{Conflict of interest statement}

The authors declare they have no conflict of interest.

\section{References}

1. Jamnongjit M, Hammes SR. Oocyte maturation: the coming of age of a germ cell. Semin Reprod Med. 2005;23:234-41; DOI:10.1055/s-2005-872451.

2. Rybska M, Knap S, Jankowski M, Jeseta M, Bukowska D. Cytoplasmic and nuclear maturation of oocytes in mammals - living in the shadow of cells developmental capability. Med J Cell Biol. 2018;1; DOI:10.2478/ acb-2018-0003.

3. Coticchio G, Dal Canto M, Renzini MM, Guglielmo MC, Brambillasca F Turchi D, Novara PV, Fadini R. Oocyte maturation: Gamete-somatic cells interactions, meiotic resumption, cytoskeletal dynamics and cytoplasmic reorganization. Hum Reprod Update. 2014; DOI:10.1093/ humupd/dmv011.

4. Watson AJ. Oocyte cytoplasmic maturation: A key mediator of oocyte and embryo developmental competence1. J Anim Sci. 2007;85:E1-3; DOI:10.2527/jas.2006-432.

5. Tanghe S, Van Soom A, Nauwynck H, Coryn M, de Kruif A. Minireview: Functions of the cumulus oophorus during oocyte maturation, ovulation, and fertilization. Mol Reprod Dev. 2002;61:414-24; DOI:10.1002/ mrd.10102.

6. Huang Z, Wells D. The human oocyte and cumulus cells relationship: new insights from the cumulus cell transcriptome. MHR Basic Sci Reprod Med. 2010;16:715-25; DOI:10.1093/molehr/gaq031.

7. Gilchrist R., Ritter L., Armstrong D. Oocyte-somatic cell interactions during follicle development in mammals. Anim Reprod Sci. 2004;8283:431-46; DOI:10.1016/j.anireprosci.2004.05.017.
8. Gilchrist RB, Lane M, Thompson JG. Oocyte-secreted factors: regulators of cumulus cell function and oocyte quality. Hum Reprod Update. 2008;14:159-77; DOI:10.1093/humupd/dmm040.

9. Regassa A, Rings F, Hoelker M, Cinar U, Tholen E, Looft C, Schellander $\mathrm{K}$, Tesfaye D. Transcriptome dynamics and molecular cross-talk between bovine oocyte and its companion cumulus cells. BMC Genomics. 2011;12; DOI:10.1186/1471-2164-12-57.

10. Tatemoto H, Sakurai N, Muto N. Protection of Porcine Oocytes Against Apoptotic Cell Death Caused by Oxidative Stress During In vitro Maturation: Role of Cumulus Cells1. Biol Reprod. 2000;63:805-10; DOI:10.1095/biolreprod63.3.805.

11. Barrett SL, Albertini DF. Cumulus cell contact during oocyte maturation in mice regulates meiotic spindle positioning and enhances developmental competence. J Assist Reprod Genet. 2010;27:29-39; DOI:10.1007/s10815-009-9376-9.

12. Dyck MK, Zhou C, Tsoi S, Grant J, Dixon WT, Foxcroft GR. Reproductive technologies and the porcine embryonic transcriptome. Anim Reprod Sci. 2014;149:11-8; DOI:10.1016/J.ANIREPROSCI.2014.05.013.

13. Lonergan P, Fair T. Maturation of Oocytes in vitro. Annu Rev Anim Biosci. 2016;4:255-68; DOI:10.1146/annurev-animal-022114-110822.

14. Li Q McKenzie LJ, Matzuk MM. Revisiting oocyte-somatic cell interactions: in search of novel intrafollicular predictors and regulators of oocyte developmental competence. Mol Hum Reprod. 2008;14:673-8; DOI:10.1093/molehr/gan064.

15. Lourenço B, Sousa AP, Almeida-Santos T, Ramalho-Santos J. Relation of cumulus cell status with single oocyte maturity, fertilization capability and patient age. J Reprod Infertil. 2014;15:15-21.

16. Janowski D, Salilew-Wondim D, Torner H, Tesfaye D, Ghanem N, Tomek W, El-Sayed A, Schellander K, Hölker M. Incidence of apoptosis and transcript abundance in bovine follicular cells is associated with the quality of the enclosed oocyte. Theriogenology. 2012;78:656-669.e5; DOI:10.1016/J.THERIOGENOLOGY.2012.03.012.

17. Walter W, Sánchez-Cabo F, Ricote M. GOplot: An R package for visually combining expression data with functional analysis. Bioinformatics. 2015;31:2912-4; DOI:10.1093/bioinformatics/btv300.

18. Tiwari M, Prasad S, Tripathi A, Pandey AN, Ali I, Singh AK, Shrivastav TG, Chaube SK. Apoptosis in mammalian oocytes: a review. Apoptosis. 2015;20:1019-25; DOI:10.1007/s10495-015-1136-y.

19. Elmore S. Apoptosis: a review of programmed cell death. Toxicol Pathol. 2007;35:495-516; DOI:10.1080/01926230701320337.

20. Kasof GM, Goyal L, White E. Btf, a novel death-promoting transcriptional repressor that interacts with Bcl-2-related proteins. Mol Cell Biol. 1999;19:4390-404; DOI:10.1128/MCB.19.6.4390.

21. Fautsch MP, Vrabel A, Subramaniam M, Hefferen TE, Spelsberg TC, Wieben ED. TGF $\beta$-inducible early gene (TIEG) also codes for early growth response a (EGR $\alpha$ ): Evidence of multiple transcripts from alternate promoters. Genomics. 1998; DOI:10.1006/geno.1998.5388.

22. Tachibana I, Imoto M, Adjei PN, Gores GJ, Subramaniam M, Spelsberg TC, Urrutia R. Overexpression of the TGFbeta-regulated zinc finger encoding gene, TIEG, induces apoptosis in pancreatic epithelial cells. J Clin Invest. 1997;99:2365-74; DOI:10.1172/JCI119418.

23. Shi Y, Vattem KM, Sood R, An J, Liang J, Stramm L, Wek RC. Identification and characterization of pancreatic eukaryotic initiation factor 2 alpha-subunit kinase, PEK, involved in translational control. Mol Cell Biol. 1998:18:7499-509.

24. Shi Y, An J, Liang J, Hayes SE, Sandusky GE, Stramm LE, Yang NN. Characterization of a mutant pancreatic eIF-2alpha kinase, PEK, and co-localization with somatostatin in islet delta cells. J Biol Chem. 1999;274:5723-30; DOI:10.1074/JBC.274.9.5723.

25. Kittler R, Putz G, Pelletier L, Poser I, Heninger A-K, Drechsel D, Fischer S, Konstantinova I, Habermann B, Grabner H, Yaspo M-L, Himmelbauer H, Korn B, Neugebauer K, Pisabarro MT, Buchholz F. An endoribonuclease-prepared siRNA screen in human cells identifies genes essential for cell division. Nature. 2004;432:1036-40; DOI:10.1038/nature03159.

26. Lin JH, Li H, Yasumura D, Cohen HR, Zhang C, Panning B, Shokat KM, LaVail MM, Walter P. IRE1 Signaling Affects Cell Fate During the Unfolded Protein Response. Science (80- ). 2007;318:944-9; DOI:10.1126/ science.1146361.

27. Suzuki H, Kanagawa H, Nishihira J. Evidence for the presence of macrophage migration inhibitory factor in murine reproductive organs and early embryos. Immunol Lett. 1996;51:141-7.

28. Wada S, Fujimoto S, Mizue Y, Nishihira J. Macrophage migration inhibitory factor in the human ovary: presence in the follicular fluids and production by granulosa cells. Biochem Mol Biol Int. 1997;41:805-14.

29. Johnson J, Espinoza T, McGaughey RW, Rawls A, Wilson-Rawls J. Notch pathway genes are expressed in mammalian ovarian follicles. Mech Dev. 2001;109:355-61; DOI:10.1016/S0925-4773(01)00523-8. 
30. Zhang C-P, Yang J-L, Zhang J, Li L, Huang L, Ji S-Y, Hu Z-Y, Gao F, Liu Y-X. Notch Signaling Is Involved in Ovarian Follicle Development by Regulating Granulosa Cell Proliferation. Endocrinology. 2011;152:2437-47; DOI:10.1210/en.2010-1182.

31. Xu J, Gridley T. Notch2 is required in somatic cells for breakdown of ovarian germ-cell nests and formation of primordial follicles. BMC Biol. 2013;11:13; DOI:10.1186/1741-7007-11-13.

32. Schlesinger TK, Bonvin C, Jarpe MB, Fanger GR, Cardinaux J-R, Johnson GL, Widmann C. Apoptosis stimulated by the 91-kDa caspase cleavage MEKK1 fragment requires translocation to soluble cellular compartments. J Biol Chem. 2002;277:10283-91; DOI:10.1074/jbc. M106885200.

33. Ou X-H, Li S, Xu B-Z, Chen L-N, Jiang M-X, Chen S-Q, Chen N-Q. Mitogen-activated protein kinase-activated protein kinase 2 is a critical regulator of pig oocyte meiotic maturation. Reprod Fertil Dev. 2015;29:22333; DOI:10.1071/RD15150.

34. Junn E, Han SH, Im JY, Yang Y, Cho EW, Um HD, Kim DK, Lee KW, Han PL, Rhee SG, Choi I. The Journal of Immunology. J Immunol. 2000;159:39218; DOI:10.4049/jimmunol.164.12.6287.

35. Wang Y, De Keulenaer GW, Lee RT. Vitamin D(3)-up-regulated protein-1 is a stress-responsive gene that regulates cardiomyocyte viability through interaction with thioredoxin. J Biol Chem. 2002;277:26496-500 DOI:10.1074/jbc.M202133200

36. Salhab M, Dhorne-Pollet S, Auclair S, Guyader-Joly C, Brisard D, Dalbies-Tran R, Dupont J, Ponsart C, Mermillod P, Uzbekova S. In vitro maturation of oocytes alters gene expression and signaling pathways in bovine cumulus cells. Mol Reprod Dev. 2013;80:166-82; DOI:10.1002/ $\operatorname{mrd} .22148$.

37. Lee S-Y, Lee H-S, Kim E-Y, Ko J-J, Yoon TK, Lee W-S, Lee K-A. Thioredoxin-Interacting Protein Regulates Glucose Metabolism and Affects Cytoplasmic Streaming in Mouse Oocytes. PLoS One. 2013;8:e70708; DOI:10.1371/journal.pone.0070708.

38. Fisher S, Gearhart JD, Oster-Granite ML. Expression of the amyloid precursor protein gene in mouse oocytes and embryos. Proc Natl Acad Sci U S A. 1991;88:1779.

39. Kimura A, Kakinuma K, Yonezawa S, Takahashi T. Expression of $\beta$-Amyloid Precursor Protein in the Porcine Ovary. Zoolog Sci. 2000;17:76977; DOI:10.2108/zsj.17.769.

40. Khan DR, Landry DA, Fournier É, Vigneault C, Blondin P, Sirard M-A Transcriptome meta-analysis of three follicular compartments and its correlation with ovarian follicle maturity and oocyte developmental competence in cows. Physiol Genomics. 2016;48:633-43; DOI:10.1152/ physiolgenomics.00050.2016. 